\section{P33 CYTOKINE AND AUTOANTIBODY PROFILES DURING TREATMENT WITH BELIMUMAB IN PATIENTS WITH SYSTEMIC LUPUS ERYTHEMATOSUS}

${ }^{1}$ Ioannis Parodis, ${ }^{1}$ Emil Åkerström, ${ }^{2}$ Christopher Sjöwall, ${ }^{3}$ Azita Sohrabian, ${ }^{4}$ Andreas Jönsen, ${ }^{1}$ Alvaro Gomez, ${ }^{2}$ Martina Frodlund, ${ }^{1}$ Agneta Zickert, ${ }^{4}$ Anders A Bengtsson, ${ }^{3} J$ ohan Rönnelid, 'Iva Gunnarsson. 'Dept. of Medicine Solna, Karolinska Institutet and Rheumatology, Karolinska University Hospital, Stockholm; ${ }^{2}$ Dept. of Clinical and Experimental Medicine, Linköping University, Linköping: ${ }^{3}$ Dept. of Immunology, Genetics and Pathology, Uppsala University, Uppsala; ${ }^{4}$ Dept. of Clinical Sciences Lund, Lund University, Lund, Sweden

\subsection{6/lupus-2020-eurolupus.8}

Background Belimumab is approved for the treatment of systemic lupus erythematosus (SLE) since 2011. We investigated whether belimumab treatment impacts on levels of cytokines and autoantibodies of interest in SLE, as well as circulating immune complexes (ICs).

Methods Longitudinally collected serum samples from 78 belimumab-treated SLE patients from the Karolinska, Skåne and Linköping University Hospitals were analysed. Serum cytokine levels and nuclear antigen autoantibody specificities were determined using addressable laser bead immunoassay, and circulating C1q-binding ICs were measured using enzyme-linked immunosorbent assay.

Results In patients with detectable levels at baseline, serum IFN- $\alpha 2$ levels were lower at month 6 (median: 8.9; IQR: 1.5-54.9 pg/mL) versus baseline (median: 28.4; IQR: 20.9-

Sustained SRI-4

\begin{tabular}{c} 
Sustained SRI-4 \\
$\mathrm{HR}(95 \% \mathrm{Cl})$ \\
\hline
\end{tabular}

\begin{tabular}{|c|c|}
\hline \multirow{7}{*}{ 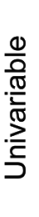 } & Age \\
\hline & Female sex \\
\hline & SLE disease duration \\
\hline & SLEDAI-2K score \\
\hline & SDI score \\
\hline & Prednisone dose \\
\hline & Low C3/C4 levels \\
\hline \multirow{13}{*}{ 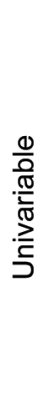 } & IFN- $\alpha 2$ \\
\hline & IL-10 \\
\hline & IL-6 \\
\hline & anti-dsDNA \\
\hline & anti-TRIM21 (SSA/Ro52) \\
\hline & anti-SSA/Ro60 \\
\hline & anti-SSB \\
\hline & anti-Sm \\
\hline & anti-Sm-RNP \\
\hline & anti-U1-RNP \\
\hline & anti-ribosomal P \\
\hline & anti-histone \\
\hline & Circulating ICs \\
\hline
\end{tabular}
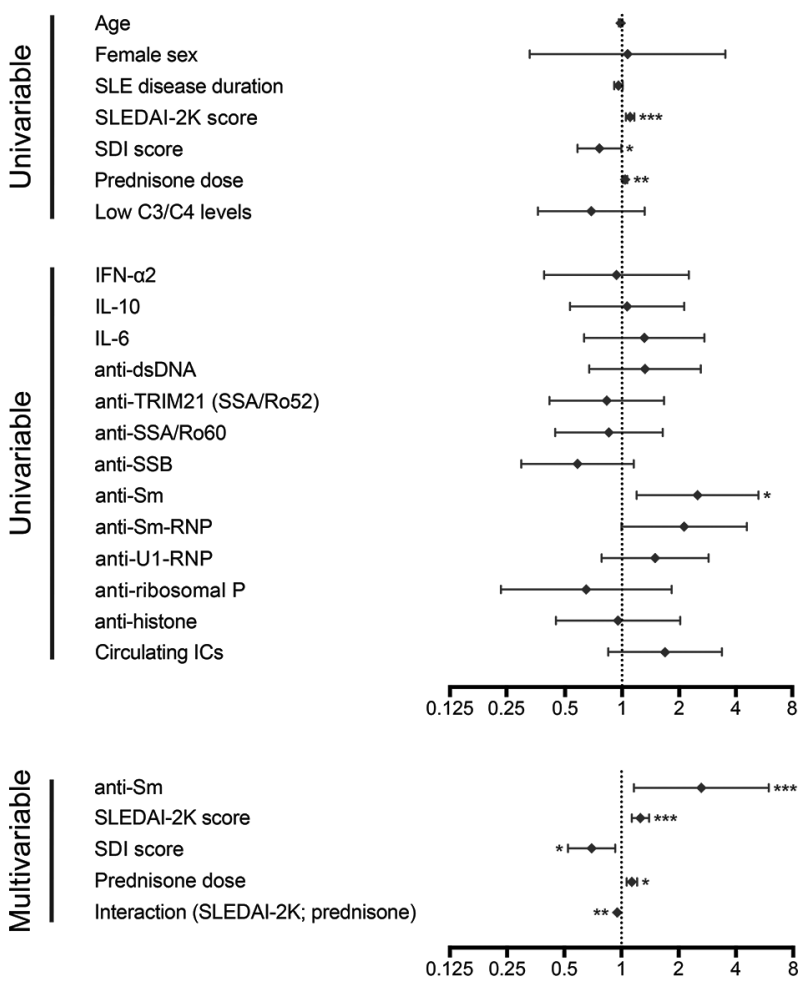

No. of patients who achieved sustained SRI-4 No. of patients in the analysis

$* \mathrm{P}<0.05 * * \mathrm{P}<0.01 * * * \mathrm{P}<0.001$
$100.3 \mathrm{pg} / \mathrm{mL} ; \mathrm{P}=0.043)$. IL-6 levels decreased from baseline (median: 7.1; IQR: $2.9-16.1 \mathrm{pg} / \mathrm{mL}$ ) to month 6 (median: 0.5 ; IQR: $0.5-6.3 \mathrm{pg} / \mathrm{mL} ; \mathrm{P}=0.018$ ) and throughout the 24 month follow-up. Levels of IL-10 (baseline median: 12.6; IQR: $2.8-29.7 \mathrm{pg} / \mathrm{mL}$ ) showed more rapid decreases from month 3 (median: 1.8; IQR: 0.6-9.1 pg/mL; $\mathrm{P}=0.003$ ). Levels of anti-dsDNA $(\mathrm{P}<0.001)$, anti-Sm $(\mathrm{P}=0.002)$, antiSmRNP $(\mathrm{P}=0.028)$, anti-U1-RNP $(\mathrm{P}<0.001)$ and anti-ribosomal $\mathrm{P}(\mathrm{P}=0.012)$ antibodies decreased from month 3 and remained decreased over the follow-up. IC levels showed decreases at month $3(\mathrm{P}=0.028), 6(\mathrm{P}=0.009)$ and 12 $(\mathrm{P}=0.021)$. Anti-Sm antibody positivity was associated with higher probability and/or shorter time to achieve sustained SLE Responder Index-4 (HR: 2.52; 95\% CI: 1.20-5.29; $\mathrm{P}=0.015$ ), independently of disease activity and other potential confounding factors (figure 1).

Conclusions In our cohort, belimumab treatment lowered IFN$\alpha 2$, IL-6, IL-10 and circulating IC levels, as well as levels of multiple autoantibodies against nuclear components. Interestingly, anti-Sm antibody positivity was associated with favourable treatment response.

\section{P34 URINARY PROTEOMICS IN LUPUS NEPHRITIS - PROSPECTIVE STUDY IN A TERTIARY CARE CENTER}

${ }^{1}$ Ramesh Ramamoorthy, ${ }^{2}$ Ragavendra, ${ }^{1}$ Kumudha Manoharan, ${ }^{1}$ Balameena Kumar. ${ }^{1}$ Institute of Rheumatology, Madras Medical College, Chennai; ${ }^{2}$ Dept. of Rheumatology, Kilpauk Medical College, Chennai, India

\subsection{6/lupus-2020-eurolupus.82}

Background Currently, major efforts have been undertaken to identify biomarkers that can predict impending lupus renal flare, development of chronic kidney disease or reflect renal histology at the time of the flare. So, this study aims to assess the correlation of urinary biomarkers MCP1 and NGAL with the disease activity in lupus nephritis (LN).

Materials and Methods This prospective study was conducted in a tertiary care center, for a period of 9 months. 60 patients with SLE were recruited. They were divided into 3 groups, 1st group with Active Lupus Nephritis $(n=22)$, 2nd group with Inactive Lupus Nephritis $(n=20)$ and 3rd group consisted of SLE patients with no renal involvement $(n=18)$. For comparison another group of age and sex matched controls was taken $(n=20)$. Disease activity was correlated with disease activity indices, baseline characteristics and biopsy. Urinary MCP1 (UMCP1) and NGAL (UNGAL) were measured. Statistical analysis using SPSS11.5 was done to find the correlation between levels of urinary biomarkers and disease activity.

Results In patients with active LN, both UMCP1/Cr and UNGAL/Cr were significantly elevated $(92.78,76.11 \mathrm{pg} / \mathrm{ml}$, $\mathrm{p}<0.001)$. In both, control group and SLE without renal involvement the values of UMCP1/Cr and UNGAL/Cr were normal $(24.44,22.22 \mathrm{pg} / \mathrm{ml}$ in control and $24.3,22.80 \mathrm{pg} / \mathrm{ml}$ in SLE without renal involvement). In patients with inactive LN the values of UMCP1/Cr and UNGAL/Cr were observed to be significantly higher than control $(44.18,38.45 \mathrm{pg} / \mathrm{ml}, \mathrm{p}<0.005)$ and lower than those of active LN. Values of UMCP1/Cr and $\mathrm{UNGAL} / \mathrm{Cr}$ were found to be in close correlation with mean rSLEDAI scores of active LN (10) and inactive LN (3.6).

Conclusions Levels of urinary biomarkers UMCP1 and UNGAL were significantly elevated in active lupus nephritis 
and found to have excellent correlation with disease activity index and rSLEDAI scores.

\section{P35 MEASURING DSDNA ANTIBODIES IN THE ELDERLY: POSITIVE RESULTS RARELY INDICATE LUPUS}

Anu Desai, Benjamin Rhodes. Rheumatology Dept., Queen Elizabeth Hospital, Birmingham, UK

\subsection{6/lupus-2020-eurolupus.83}

Background Antibodies to dsDNA are reported to be specific for lupus and are included in the main classification criteria, although pitfalls when used as a 'screening' test in patients with multiple medical symptoms have been described. Lupus is typically diagnosed in the first 50 years of life and clinically active disease is unusual in the elderly. We evaluated dsDNA requests made in elderly patients ( $>80$ years) in our centre.

Methods Review of 23,287 consecutive dsDNA antibody requests made through the University of Birmingham Immunology Lab between 2009 and 2019. Review of available clinical records for patients with positive results.

Results 993 dsDNA antibody requests were made for patients $>80$ years old (mean 84.2 years), representing $0.04 \%$ of total requests. 578 were female, 411 were male and 5 unknown. 138 (13.9\%) requests were made from primary care, $80(8.0 \%)$ from the critical care/emergency department, $623(62.5 \%)$ from medical wards and outpatients and 97 (4.4\%) from rheumatology. The remainder were from neighbouring hospitals. 67 patients (6.7\%) tested positive for dsDNA by ELISA (lab normal range) and 31 (3.1\%) by Crithidia.

Clinical records were available for 58 patients with positive dsDNA antibodies. Three were known to have longstanding SLE. No patients were diagnosed as having SLE at or after the time of dsDNA antibody testing. Sixteen patients had other autoimmune diseases including rheumatoid arthritis, Sjogrens Syndrome, myasthenia gravis, microscopic polyangiitis, polymyalgia rheumatica, bullous pemphigoid and immune thrombocytopenia. Four patients had underlying malignancy (one each of lung, prostate, bladder and colon). The remainder had a mix of infections and general medical presentations.

One hundred randomly selected patients with negative dsDNA antibody were reviewed and no cases of lupus were identified, leading to a calculated specificity of dsDNA antibodies for lupus of only $64.5 \%$.

Conclusions Antibodies to dsDNA are not uncommon in unwell elderly patients presenting to hospital or primary care. They are rarely indicative of SLE. The reported high specificity of dsDNA antibodies for lupus are not applicable in this age group.

\section{P36 USING MACHINE LEARNING TO IDENTIFY AND STRATIFY PATIENTS WITH JUVENILE-ONSET SLE}

George Robinson, Junjie Peng, Anna Radziszewska, Chris Wincup, Hannah Peckham, Meena Naja, David Isenberg, Yiannis Ioannou, Ines Pineda-Torra, Coziana Ciurtin, Elizabeth Jury. Dept. of Medicine, University College London, London, UK

10.1136/lupus-2020-eurolupus.84
Background Juvenile-onset Systemic Lupus Erythematosus (JSLE) is a complex disease characterised by diagnosis and treatment delays. We applied a machine learning (ML) approach to explore new diagnostic signatures for JSLE based on immune-phenotyping data.

Methods Immune-phenotyping of 28 T-cell, B-cell and myeloid-cell subsets in 67 age and sex-matched JSLE patients and 39 healthy controls (HCs) was performed by flow cytometry. A balanced random forest ML predictive model was developed $(10,000$ decision trees). $75 \%$ of sample data was randomly selected as a training set, the remaining $25 \%$ out-of-bag data was used for validation. Reciever operator characteristic, 10fold cross validation, Sparse Partial Least Squares-Discriminant Analysis (sPLS-DA) and linear regression was used to validate the model.

Results In JSLE, a global change in immunological architecture was established compared to HCs: many of the immune cell relationships identified in HCs using correlation comparison analysis were inverted or exacerbated in JSLE, including significantly inverted correlations between intermediate monocytes and memory B-cell populations and CD4/CD8 memory T-cells and B-cell memory in SLE versus HCs. Using immune-phenotyping data a ML model was developed and validated (accuracy $=87.80 \%$ ) showing that JSLE patients could be distinguished from HCs with high confidence using immunological parameters. The top variables contributing to the model included $\mathrm{CD} 19^{+}$unswitched memory B-cells, naïve B-cells, CD14 ${ }^{+}$monocytes and memory T-cell subsets. The 'JSLE immune signature' was also sucessfully validated using sPLS-DA and linear regression. To assess whether the validated signature could be used to further stratify JSLE patients, K-mean clustering was applied. Four JSLE groups each with a distinct immune and clinical profile were identified. Finally, network analysis identified specific clinical features associated with each of the top JSLE immune-signature variables.

Conclusion Using a combined ML approach, a distinct immune signature was identified that discriminated between JSLE patients and HCs and further stratified patients. This signature could have diagnostic and therapeutic implications.

\section{P37 NON-INVASIVE BIOMARKERS OF DISEASE ACTIVITY IN SYSTEMIC LUPUS ERYTHEMATOSUS}

${ }^{1,2}$ Guillermo Ruacho, ${ }^{2}$ Ronaldo Lira-Junior, ${ }^{1}$ Iva Gunnarsson, ${ }^{1}$ Elisabet Svenungsson, ${ }^{2}$ Elisabeth Almer Boström. 'Unit of Rheumatology, Dept. of Medicine, Karolinska University Hospital, Stockholm; ${ }^{2}$ Division of Oral Diseases, Dept. of Dental Medicine. Karolinska Institutet, Stockholm, Sweden

\subsection{6/lupus-2020-eurolupus.85}

Purpose The possibility of exploring other body fluids to identify potential biomarkers of systemic inflammation has emerged as a non-invasive alternative to blood samples for both diagnosis and control of disease activity. We investigated the levels of innate-immunity related biomarkers in saliva, serum, and urine from Systemic Lupus Erythematous (SLE) patients and their correlation to each other and to disease activity.

Methods We included 84 SLE patients and 20 controls from the general population, all participants underwent a thorough clinical examination. Disease activity as measured with the Systemic Lupus Activity Measure (SLAM) and SLE Disease 